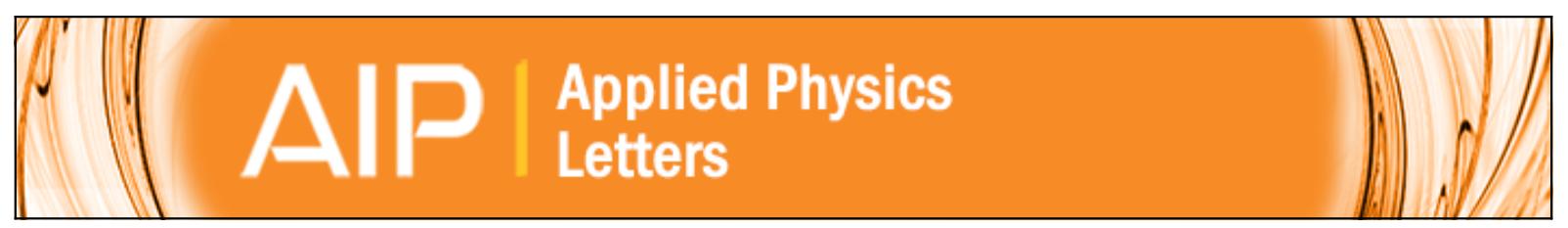

\title{
Probing magnetic and electric optical responses of silicon nanoparticles
}

Dmitry Permyakov, Ivan Sinev, Dmitry Markovich, Pavel Ginzburg, Anton Samusev, Pavel Belov, Vytautas Valuckas, Arseniy I. Kuznetsov, Boris S. Luk'yanchuk, Andrey E. Miroshnichenko, Dragomir N. Neshev, and Yuri S. Kivshar

Citation: Applied Physics Letters 106, 171110 (2015); doi: 10.1063/1.4919536

View online: http://dx.doi.org/10.1063/1.4919536

View Table of Contents: http://scitation.aip.org/content/aip/journal/apl/106/17?ver=pdfcov

Published by the AIP Publishing

\section{Articles you may be interested in}

Nanometer-resolution optical probe using a metallic-nanoparticle-intercalated carbon nanotube

J. Appl. Phys. 109, 013110 (2011); 10.1063/1.3511437

Invited Review Article: A review of techniques for attaching micro- and nanoparticles to a probe's tip for surface force and near-field optical measurements

Rev. Sci. Instrum. 78, 081101 (2007); 10.1063/1.2754076

Optical probing of a silicon integrated circuit using electric-field-induced second-harmonic generation Appl. Phys. Lett. 88, 114107 (2006); 10.1063/1.2180446

Dynamic optical probing of the magnetic anisotropy of nickel-ferrite nanoparticles

J. Appl. Phys. 96, 5226 (2004); 10.1063/1.1790574

In-plane magnetization of garnet films imaged by proximal probe nonlinear magneto-optical microscopy J. Appl. Phys. 88, 2166 (2000); 10.1063/1.1302734

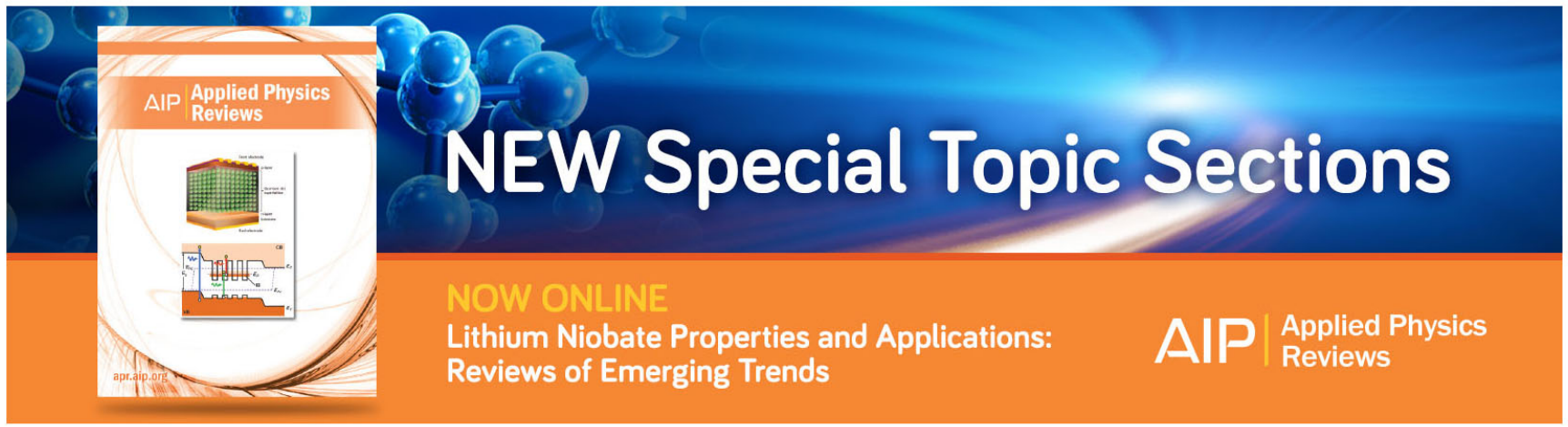




\title{
Probing magnetic and electric optical responses of silicon nanoparticles
}

\author{
Dmitry Permyakov, ${ }^{1}$ Ivan Sinev, ${ }^{1}$ Dmitry Markovich, ${ }^{1}$ Pavel Ginzburg, ${ }^{1,2}$ Anton Samusev, ${ }^{1}$ \\ Pavel Belov, ${ }^{1}$ Vytautas Valuckas, ${ }^{3,4}$ Arseniy I. Kuznetsov, ${ }^{3}$ Boris S. Luk'yanchuk, ${ }^{3}$ \\ Andrey E. Miroshnichenko, ${ }^{5}$ Dragomir N. Neshev, ${ }^{5}$ and Yuri S. Kivshar ${ }^{1,5}$ \\ ${ }^{1}$ ITMO University, St. Petersburg 197101, Russia \\ ${ }^{2}$ School of Electrical Engineering, Tel Aviv University, Ramat Aviv, Tel Aviv 69978, Israel \\ ${ }^{3}$ Data Storage Institute, A*STAR (Agency for Science Technology and Research), Singapore 117608 \\ ${ }^{4}$ Department of Electrical and Computer Engineering, National University of Singapore, Singapore 117576 \\ ${ }^{5}$ Nonlinear Physics Centre, Research School of Physics and Engineering, Australian National University, \\ Canberra ACT 2601, Australia
}

(Received 6 March 2015; accepted 20 April 2015; published online 29 April 2015)

\begin{abstract}
We study experimentally both magnetic and electric optically induced resonances of silicon nanoparticles by combining polarization-resolved dark-field spectroscopy and near-field scanning optical microscopy measurements. We reveal that the scattering spectra exhibit strong sensitivity of electric dipole response to the probing beam polarization and attribute the characteristic asymmetry of measured near-field patterns to the excitation of a magnetic dipole mode. The proposed experimental approach can serve as a powerful tool for the study of photonic nanostructures possessing both electric and magnetic optical responses. (C) 2015 AIP Publishing LLC.

[http://dx.doi.org/10.1063/1.4919536]
\end{abstract}

In recent years, dielectric nanostructures composed of materials with high refractive index have been studied intensively as promising building blocks for all-dielectric nanophotonic devices. ${ }^{1-10}$ While applications of plasmonic components are limited by a number of factors including high ohmic losses, ${ }^{11}$ high-index dielectric nanostructures are practically free from losses, and they can also exhibit optically induced magnetic responses in the visible range bringing an additional degree of freedom for light manipulation at the nanoscale. Coexistence of tunable magnetic and electric responses ${ }^{4-6}$ in high-index nanoparticles at optical frequencies triggers the development of concepts for light-manipulating components including impedance-matched metasurfaces, ${ }^{12}$ superdirective antennas, ${ }^{13}$ and precise control over nanoparticle scattering, including zero back-scattering. ${ }^{6-9}$

A basic nanostructure, which can exhibit both electric and magnetic responses at optical frequencies, is a highindex dielectric nanosphere. ${ }^{1-6,8}$ Magnetic scattering resonances in the visible range were observed for nanoparticles made of silicon ${ }^{4,5}$ and gallium arsenide. ${ }^{7}$

The most common way to characterize the optical properties of a single nanoparticle is to measure its scattering spectra. The experimental results for the scattering spectra of a single nanosphere are usually in a good agreement with the predictions of Mie theory. ${ }^{4-6,14}$ The nature of observed features in the scattering spectra can be interpreted via numerical multipolar decomposition method, allowing to distinguish the contributions of dipole and higher-order multipole modes and resolve intriguing phenomena such as excitation of toroidal or anapole modes. ${ }^{10,15,16}$ Otherwise, scattering near-field scanning optical microscopy technique ${ }^{17}$ can be employed for direct observation of high-order multipole modes in single dielectric nanostructures. ${ }^{18}$

In this letter, we make the next step in the study of optically induced magnetic resonances of nanostructures and propose an alternative experimental approach allowing to distinguish the optical magnetic dipole (MD) and electric dipole (ED) resonances directly from experiment. This is made possible by combining the polarization-resolved darkfield spectroscopy and near-field scanning optical microscopy (NSOM) measurements. We believe that the proposed experimental approach can serve as a powerful tool for the study of photonic nanostructures possessing both electric and magnetic optical responses.

Silicon $(\mathrm{Si})$ nanoparticles of various sizes are fabricated by femtosecond laser ablation of a Si wafer as described in Ref. 6. The laser beam passes through the glass substrate placed on the top of the wafer and ablates the Si surface. The ablated silicon nanoparticles are deposited and solidified on the glass substrate. Then the nanoparticles of different sizes are imaged using dark-field optical microscope and scanning electron microscope (SEM).

Two experimental setups used to characterize the optical responses of single nanoparticles are sketched in Fig. 1. To study the scattering properties of Si nanoparticles as a function of wavelength and polarization of the incident beam, we use a home-made dark-field microscope with independent excitation (side) and collection (upper) optical channels, Fig. 1(a). Polarized white light is weakly focused with an objective (Mitutoyo MPlanApo $10 \times, \mathrm{NA}=0.28$ ) on the sample surface at oblique incidence $\left(25^{\circ}\right.$ to the sample surface). The light scattered by a single particle is collected by the second objective (Mitutoyo MPlanApo 50×, $\mathrm{NA}=0.55)$ and analyzed with a spectrometer in confocal arrangement. Similar experimental method was used for the characterization of optical response of plasmonic nanostructures in Refs. 19 and 20.

To obtain information on the spatial distribution of the electromagnetic field in the vicinity of the particle, we use the aperture-type NSOM instrument (AIST-NT, Inc., tapered aluminium-coated fiber probe) operating in the collection mode, ${ }^{21}$ Fig. 1(b). For sample excitation, we employ a 
(a)

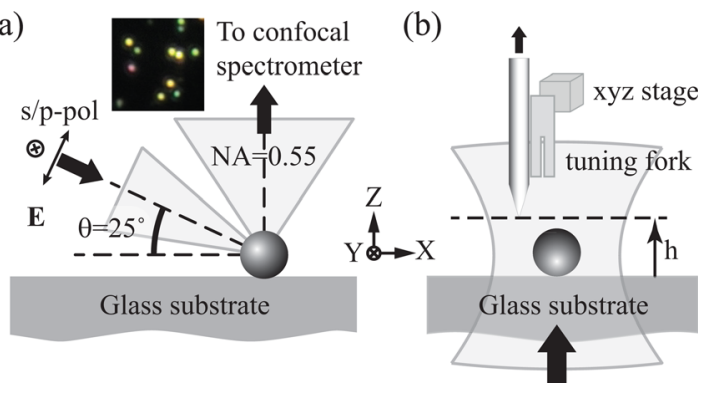

FIG. 1. (a) Experimental setup for the polarization-resolved dark-field spectroscopy. A sample is excited by polarized white light from the side. Scattered light is collected from the top. The inset shows dark-field image of the sample $\left(10 \times 10 \mu \mathrm{m}^{2}\right)$. (b) Schematic of NSOM experimental setup. Si particle is excited from the bottom by a weakly focused supercontinuum laser beam with tunable central wavelength.

supercontinuum source (Fianium WhiteLase SC400-6) combined with the tunable bandpass filter (Fianium SuperChrome) yielding a beam with a spectral width of approximately $10 \mathrm{~nm}$ with tunable central wavelength. After the filtering, the beam is polarized with a Glan prism and weakly focused on the sample surface through the substrate by an achromatic doublet lens (focal distance $\mathrm{f}=5 \mathrm{~cm}$ ) to a spot with the diameter of approximately $30 \mu \mathrm{m}$, Fig. 1(b). The scanning is performed in constant-height mode ${ }^{21}$ (height $h \approx 200 \mathrm{~nm}$ above the substrate surface) by using capacitive sensor feedback loop.

To gain further insight into the nature of the observed peaks as compared to previously reported results on unpolarized dark-field spectroscopy, ${ }^{4,5}$ we improved the setup by introducing a polarizer to the excitation channel, thus achieving polarization resolution. Since in our dark-field spectroscopy setup (see Fig. 1(a)), we use relatively low numerical aperture objective $(\mathrm{NA}=0.55)$ in the collection channel, the scattering from the $\mathrm{x}$ - and $\mathrm{y}$-projections of the overall dipole moments induced in the nanoparticle dominate in the collected signal. (This follows from the fact that the point-like dipole emission is suppressed along the direction of the dipole itself. ${ }^{22}$ ) Accordingly, we can expect the collected ED response to be stronger for s-polarized rather than for p-polarized excitation and vice versa for the MD resonance, which is indeed true for scattering in free space (see scattering cross-section simulations in Fig. 2(c), dashed lines). Note that large angles of incidence are beneficial since they ensure bigger difference between the magnitudes of induced inplane dipoles moments for s- and p-polarizations.

The dark-field spectra obtained from a single Si nanoparticle for s- and p-polarized incident beam are shown in Fig. 2(b). In order to verify the experimental results, we calculated the scattering cross-sections (SCS) for Si nanoparticle on a glass (refractive index 1.5) substrate using analytical solution. ${ }^{23}$ The diameter of the sphere was set to be $150 \mathrm{~nm}$, as it is estimated from the SEM image shown in the inset to Fig. 2(b). The crystalline silicon dielectric permittivity model used in the simulations was taken from Ref. 24 . The sphere was illuminated by a linearly polarized plane wave at oblique incidence ( $25^{\circ}$ to the sample surface, same as in the experiment). The collected signal was modelled by integration of the scattered radiation within the cone with half-angle of $33.4^{\circ}$, which corresponds to the collection objective NA of 0.55 . The scattering spectra calculated in such manner for both excitation polarizations are presented in Fig. 2(c) (solid lines) and demonstrate excellent agreement with the experimental curves (Fig. 2(b)).

Comparing s- and p-polarized spectra obtained both experimentally and numerically, one can immediately see that the feature corresponding to ED resonance exhibits the expected polarization sensitivity. The peak becomes pronounced for s-polarization and is suppressed for p-polarization. On the contrary, MD resonance does not demonstrate such polarization dependence.

To provide even better distinction between the magnetic and electric dipole contributions, we complemented our darkfield measurements with NSOM experiments performed at $\mathrm{ED}$ and MD resonant wavelengths. Importantly, NSOM is known to have different sensitivities to different components of the electromagnetic field. ${ }^{25-28}$ Moreover, recent papers ${ }^{29,30}$ provide experimental evidence that NSOM signal can be interpreted in terms of lateral (perpendicular to the probe axis) electromagnetic field components, while in Ref. 31 we have shown that the maps of lateral magnetic or electric field measured above a point-like dipole demonstrate different symmetry for electric and magnetic dipoles. This makes NSOM a promising tool for distinguishing the magnetic and electric origin of dipole responses of nanoscale systems.

In order to check the possibility to distinguish electric and magnetic responses of $\mathrm{Si}$ nanoparticle in NSOM
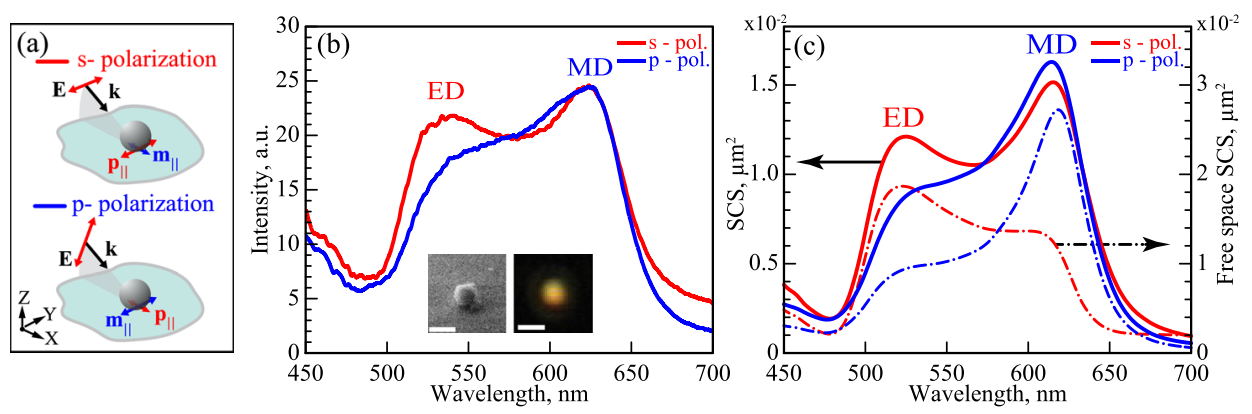

FIG. 2. (a) Sketch of the electric field of s- and p-polarized incident wave in dark-field experimental geometry. The dominating lateral dipole moments of a nanoparticle for both cases are shown. (b) Experimental dark-field scattering spectra of a nanoparticle with diameter $d \approx 150 \mathrm{~nm}$ obtained for s- and ppolarized incident beam. The images of the particle obtained using SEM (angle of view $50^{\circ}$, scale bar $200 \mathrm{~nm}$ ) and dark-field microscope (scale bar $1 \mu \mathrm{m}$ ) are shown in the left and right insets, respectively. (c) Simulated scattering cross section spectra calculated for a spherical silicon nanoparticle $(d=150 \mathrm{~nm})$ on a glass substrate (solid lines) and in free space (dashed lines). 
ED

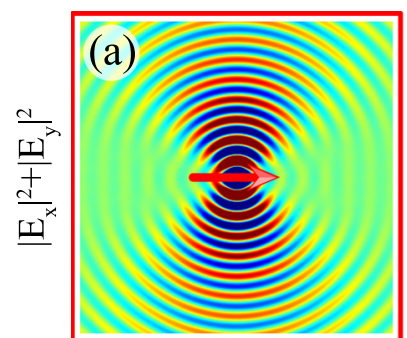

(c)

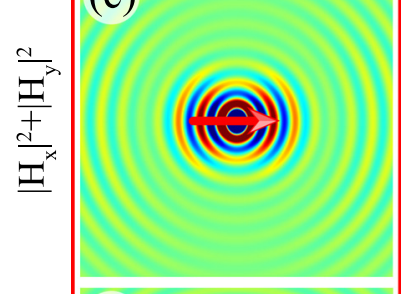

(e)

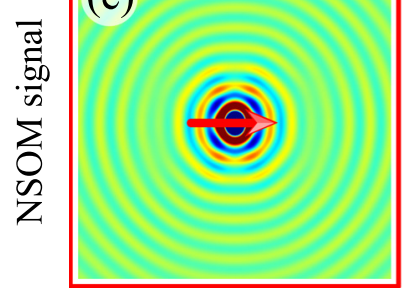

MD

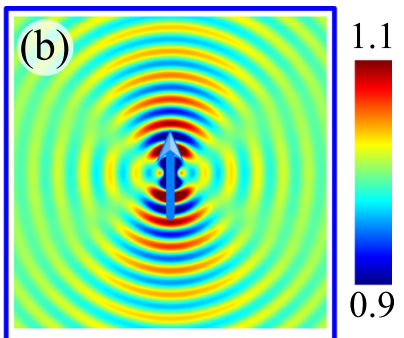

(d)

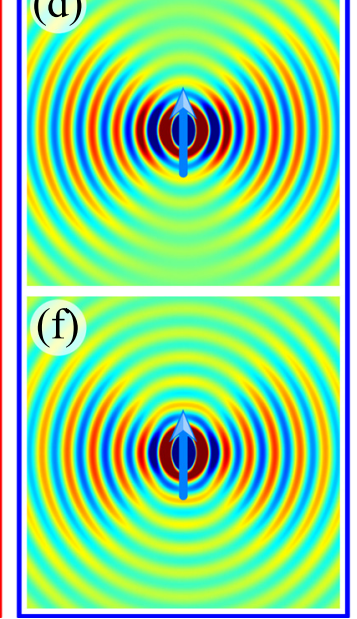

FIG. 3. Numerical results for Si sphere with the diameter of $d=150 \mathrm{~nm}$ excited by plane wave at the wavelengths of electric $(\lambda=510 \mathrm{~nm}$, left column $)$ and magnetic ( $\lambda=610 \mathrm{~nm}$, right column) dipole resonances. The maps of in-plane electric (a) and (b), in-plane magnetic (c) and (d) field intensities and calculated NSOM signal picked up by a probe with the aperture of $200 \mathrm{~nm}(\mathrm{e})$ and (f) at the elevation of $50 \mathrm{~nm}$ above the nanoparticle. The size of each image is $8 \times 8 \mu \mathrm{m}^{2}$.

experiments, we performed full-wave electromagnetic field simulations using finite-difference time-domain method (Lumerical FDTD Solutions). In the simulations, the particle was excited by a plane wave propagating along the $\mathrm{z}$-direction, the field monitor was placed at $50 \mathrm{~nm}$ above the silicon sphere. We also ensured that the change of the angle of incidence did not take strong effect on the scattering resonances' frequencies

The simulated patterns of the lateral electric and magnetic field intensities at the wavelengths of ED and MD resonances are shown in Figs. 3(a)-3(d). The observed intensity modulation originates from the interference of the radiation scattered by the particle with the incident plane wave. A closer look reveals that these maps demonstrate different "degree of asymmetry" (DOA). Defined as the ratio between magnitude of interference pattern modulation along and perpendicular to polarization of incident beam, DOA can be used to quantitatively characterize the contribution of certain dipole (either ED or MD) to the scattering of the particle. As discussed in Ref. 31, only the presence of MD response can lead to asymmetry in lateral magnetic fields, while asymmetry in electric field map is a signature of ED contribution. Since all maps (Fig. 3(a)-3(d)) demonstrate azimuthal angle dependence, we can conclude that the nanoparticle possesses both electric and magnetic responses at the selected wavelengths. However, while the "main" (resonant) dipole contributions
(ED in Fig. 3(a) and MD in Fig. 3(d)) are equally strong, the comparison of DOA in Figs. 3(c) and 3(b) shows that the "secondary" dipole contribution is stronger at the MD resonance wavelength (ED response at MD resonance is stronger than MD response at ED resonance). This is in good agreement with previous results of multipole decomposition reported elsewhere, ${ }^{1,5}$ which demonstrate that ED response is spectrally broader than MD response.

To understand whether the asymmetry related to the contributions of dipole responses can indeed manifest itself in NSOM experiments, we performed the reconstruction of near-field signal from rigorous FDTD simulations. To account for the sensitivity of the NSOM probe to different electromagnetic field components, we applied a method based on the electromagnetic reciprocity theorem. ${ }^{30,32}$ The maps of NSOM signal simulated in such manner for tip aperture size of $200 \mathrm{~nm}$ are shown in Figs. 3(e) and 3(f) for the wavelengths of ED and MD, respectively. Though the model takes into account both electric and magnetic fields in the vicinity of the sample, the NSOM maps closely resemble the patterns of the in-plane magnetic fields, especially at some distance from the nanoparticle (Figs. 3(e) and 3(f)). This indicates that for the considered experimental geometry the aperture-type optical fiber NSOM probe acts as a "magnetic analyzer" collecting mainly lateral magnetic fields. ${ }^{27,28}$

The results of the NSOM experiments performed at the wavelengths of ED and MD resonances are shown in Fig. 4. The obtained NSOM maps closely match the numerical simulations (Figs. 3(e) and 3(f)). In the case of ED resonance, the NSOM pattern does not demonstrate strong dependence on azimuthal angle, indicating weak magnetic response at this wavelength. This is best illustrated by radial NSOM map profiles, which were obtained by averaging the radial sections of the maps within $45^{\circ}$ sector along and across the polarization direction (see Fig. 4(c) for ED map profile). On the contrary,
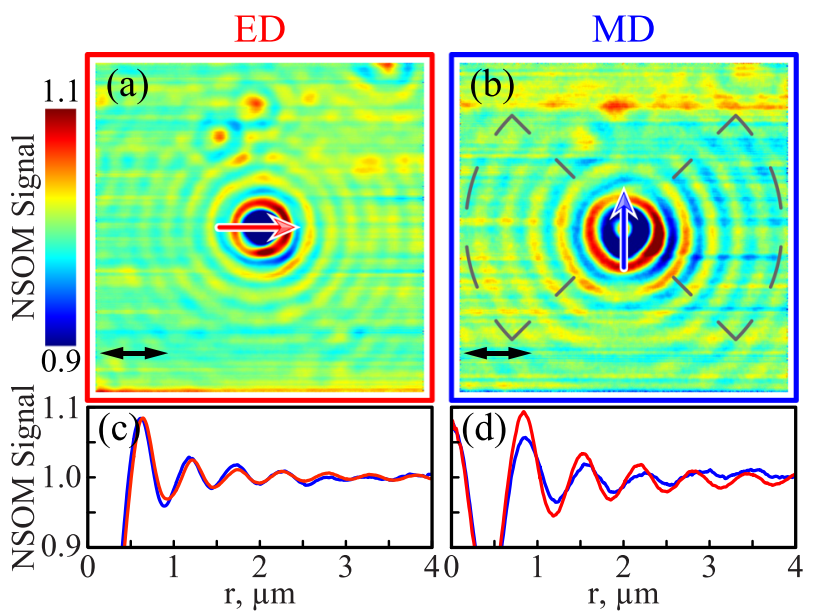

FIG. 4. (a) and (b) NSOM scans of Si nanosphere at the wavelengths of ED $(\lambda=510 \mathrm{~nm},(\mathrm{a}))$ and $\mathrm{MD}(\lambda=610 \mathrm{~nm}$, (b)) resonances. Red and blue arrows in the center of each plot illustrate the induced electric and magnetic dipole moments, respectively. The size of each image is $8 \times 8 \mu \mathrm{m}^{2}$. Dashed lines on panel (b) mark the sectors with maximum interference pattern modulation. (c) and (d) Respective averaged radial profiles of NSOM maps for ED (c) and MD (d) wavelengths showing the magnitude of signal modulation along (red line) and across (blue line) the polarization direction (indicated by a black arrow in the top row panels). 
at the MD resonance the NSOM signal (Fig. 4(b)) shows distinguishable asymmetry with the lobes aligned along the incident beam polarization (Fig. 4(d)), confirming the magnetic nature of the observed scattering resonance.

In conclusion, we have studied, both experimentally and numerically, the scattering properties of an isolated silicon nanosphere. We have employed an experimental approach which comprises polarization-resolved dark-field spectroscopy and near-field scanning optical microscopy. Polarizationresolved dark-field spectroscopy allows to determine spectral positions of optical scattering resonances and demonstrates strong sensitivity of electric dipole response to the incident polarization. In turn, the magnetic dipole response of the nanoparticle can be directly identified through the asymmetry in the NSOM patterns obtained using an aperture-type probe operating as an effective magnetic field analyzer.

The presented results confirm that the proposed experimental approach allows for discrimination of the dipole optical responses of both magnetic and electric nature. Importantly, this can be done directly from the experimental data without employing complementary numerical simulations.

This work was financially supported by Government of Russian Federation (Project Nos. 14.584.21.0009 10 and GOSZADANIE 2014/190, Zadanie No. 3.561.2014/K, 074U01), Russian Foundation for Basic Research and the Australian Research Council. The authors at DSI were supported by DSI core funds. We acknowledge useful discussions with L. (Kobus) Kuipers and A. Krasnok and thank I. Mukhin for SEM characterization.

${ }^{1}$ A. B. Evlyukhin, C. Reinhardt, A. Seidel, B. S. Luk'yanchuk, and B. N. Chichkov, Phys. Rev. B 82, 045404 (2010).

${ }^{2}$ A. García-Etxarri, R. Gómez-Medina, L. S. Froufe-Pérez, C. López, L. Chantada, F. Scheffold, J. Aizpurua, M. Nieto-Vesperinas, and J. Sáenz, Opt. Express 19, 4815 (2011)

${ }^{3}$ A. E. Krasnok, A. E. Miroshnichenko, P. A. Belov, and Y. S. Kivshar, Opt. Express 20, 20599 (2012).

${ }^{4}$ A. I. Kuznetsov, A. E. Miroshnichenko, Y. H. Fu, J. Zhang, and B. Luk'yanchuk, Sci. Rep. 2, 492 (2012).

${ }^{5}$ A. B. Evlyukhin, S. M. Novikov, U. Zywietz, R. L. Eriksen, C. Reinhardt, S. I. Bozhevolnyi, and B. N. Chichkov, Nano Lett. 12, 3749 (2012).

${ }^{6}$ Y. H. Fu, A. I. Kuznetsov, A. E. Miroshnichenko, Y. F. Yu, and B. Luk'yanchuk, Nat. Commun. 4, 1527 (2013).

${ }^{7}$ S. Person, M. Jain, Z. Lapin, J. J. Sáenz, G. Wicks, and L. Novotny, Nano Lett. 13, 1806 (2013).
${ }^{8}$ P. Albella, M. A. Poyli, M. K. Schmidt, S. A. Maier, F. Moreno, J. J. Sáenz, and J. Aizpurua, J. Phys. Chem. C 117, 13573 (2013).

${ }^{9}$ B. S. Lukyanchuk, N. V. Voshchinnikov, R. Paniagua-Dominguez, and A. I. Kuznetsov, "Optimum forward light scattering by spherical and spheroidal dielectric nanoparticles with high refractive index," preprint arXiv:1412.2861 (2014)

${ }^{10}$ R. M. Bakker, D. Permyakov, Y. F. Yu, D. Markovich, R. PaniaguaDomínguez, L. Gonzaga, A. Samusev, Y. S. Kivshar, B. Lukyanchuk, and A. I. Kuznetsov, Nano Lett. 15, 2137 (2015).

${ }^{11}$ D. K. Gramotnev and S. I. Bozhevolnyi, Nat. Photonics 4, 83 (2010).

${ }^{12}$ M. Decker, I. Staude, M. Falkner, J. Dominguez, D. N. Neshev, I. Brener, T. Pertsch, and Y. S. Kivshar, "High-efficiency dielectric Huygens' surfaces," Adv. Opt. Mater. (published online).

${ }^{13}$ A. E. Krasnok, C. R. Simovski, P. A. Belov, and Y. S. Kivshar, Nanoscale 6, 7354 (2014).

${ }^{14}$ J.-M. Geffrin, B. García-Cámara, R. Gómez-Medina, P. Albella, L. Froufe-Pérez, C. Eyraud, A. Litman, R. Vaillon, F. González, M. NietoVesperinas et al., Nat. Commun. 3, 1171 (2012).

${ }^{15}$ A. I. Kuznetsov, A. E. Miroshnichenko, Y. H. Fu, V. Viswanathan, M. Rahmani, V. Valuckas, Z. Y. Pan, Y. Kivshar, D. S. Pickard, and B. Luk'yanchuk, Nat. Commun. 5, 3104 (2014).

${ }^{16}$ A. E. Miroshnichenko, A. B. Evlyukhin, Y. F. Yu, R. M. Bakker, A. Chipouline, A. I. Kuznetsov, B. Lukyanchuk, B. N. Chichkov, and Y. S. Kivshar, "Seeing the unseen: Observation of an anapole with dielectric nanoparticles," preprint arXiv:1412.0299 (2014).

${ }^{17}$ N. Ocelic, A. Huber, and R. Hillenbrand, Appl. Phys. Lett. 89, 101124 (2006).

${ }^{18}$ T. G. Habteyes, I. Staude, K. E. Chong, J. Dominguez, M. Decker, A. Miroshnichenko, Y. Kivshar, and I. Brener, ACS Photonics 1, 794 (2014).

${ }^{19}$ M. W. Knight, J. Fan, F. Capasso, and N. J. Halas, Opt. Express 18, 2579 (2010).

${ }^{20}$ J. A. Fan, K. Bao, J. B. Lassiter, J. Bao, N. J. Halas, P. Nordlander, and F. Capasso, Nano Lett. 12, 2817 (2012).

${ }^{21}$ J.-J. Greffet and R. Carminati, Prog. Surf. Sci. 56, 133 (1997).

${ }^{22}$ J. D. Jackson, Classical Electrodynamics (Wiley, New York, 1998).

${ }^{23}$ P. Bobbert and J. Vlieger, Physica A 137, 209 (1986).

${ }^{24}$ E. D. Palik, Handbook of Optical Constants of Solids (Academic Press, 1985).

${ }^{25}$ M. Burresi, D. van Oosten, T. Kampfrath, H. Schoenmaker, R. Heideman, A. Leinse, and L. Kuipers, Science 326, 550 (2009).

${ }^{26}$ D. C. Kohlgraf-Owens, S. Sukhov, and A. Dogariu, Opt. Lett. 37, 3606 (2012).

${ }^{27}$ H. Kihm, S. Koo, Q. Kim, K. Bao, J. Kihm, W. Bak, S. Eah, C. Lienau, H. Kim, P. Nordlander, N. Halas, N. Park, and D.-S. Kim, Nat. Commun. 2, 451 (2011).

${ }^{28}$ H. W. Kihm, J. Kim, S. Koo, J. Ahn, K. Ahn, K. Lee, N. Park, and D.-S. Kim, Opt. Express 21, 5625 (2013).

${ }^{29}$ B. Le Feber, N. Rotenberg, D. Beggs, and L. Kuipers, Nat. Photonics 8, 43 (2014).

${ }^{30}$ I. S. Sinev, P. M. Voroshilov, I. S. Mukhin, A. I. Denisyuk, M. E. Guzhva, A. K. Samusev, P. A. Belov, and C. R. Simovski, Nanoscale 7, 765 (2015).

${ }^{31}$ D. Permyakov, I. Mukhin, I. Shishkin, A. Samusev, P. Belov, and Y. Kivshar, JETP Lett. 99, 622 (2014)

${ }^{32}$ J. Porto, R. Carminati, and J.-J. Greffet, J. Appl. Phys. 88, 4845 (2000). 